EOMmUn: Communication et organisation

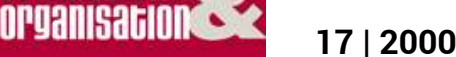

Europe et communication

\title{
Culture et télévision en Europe : le cas d'Arte
}

Nahima Vianna

\section{OpenEdition}

Journals

Édition électronique

URL : http://journals.openedition.org/communicationorganisation/2376

DOI : 10.4000/communicationorganisation.2376

ISSN : 1775-3546

Éditeur

Presses universitaires de Bordeaux

Édition imprimée

Date de publication : 1 mai 2000

ISSN : 1168-5549

\section{Référence électronique}

Nahima Vianna, "Culture et télévision en Europe : le cas d'Arte », Communication et organisation [En ligne], 17 | 2000, mis en ligne le 27 mars 2012, consulté le 01 mai 2019. URL : http://

journals.openedition.org/communicationorganisation/2376; DOI : 10.4000/

communicationorganisation.2376

Ce document a été généré automatiquement le 1 mai 2019.

(c) Presses universitaires de Bordeaux 


\title{
Culture et télévision en Europe : le cas d'Arte
}

\author{
Nahima Vianna
}

En 1980, la France signe avec l'Allemagne une convention pour mener les recherches qui auront comme résultat la mise en orbite, par le lanceur Ariane, de TV Satl et de TDF1. Ce dernier est évalué, à l'époque, comme capable d'atteindre un public de 400 millions de personnes réparties par toute l'Europe, la Méditerranée et même le nord de l'Afrique, ce qui représentait un enjeu considérable puisque le parc télévisuel européen se trouvait encombré dans les bandes de fréquences hertziennes : la demande du marché audiovisuel ne pouvait plus être satisfaite car les canaux de diffusion dépassaient la capacité de transmission terrestre. Le satellite apporte donc à la France la possibilité d'insertion dans le marché audiovisuel mondial, jusqu'à présent dominé par les grand groupes américains. Le scénario audiovisuel européen de cette époque est celui d'une télévision commerciale émergente et fragile, dans un marché encore peu organisé, ne peut faire face à l'arrivée des produits dit mondialisés provenant de l'Amérique du Nord. En 1987, les États-Unis devenaient les principaux producteurs de programmes audiovisuels, avec $70 \%$ des exportations mondiales, en détriment de l'Europe, qui elle, figurait dans ce paysage avec l'achat de $40 \%$ des programmes produits par les États-Unis. Ce paysage constituait une mosaïque de facteurs qui donneront naissance à la première chaîne culturelle européenne gérée par deux des plus grands pays de l'Europe. Arte - ou Association Relative à la Télévision Européenne - était le produit de trois logiques interactives bien conduites par l'initiative d'un État Français soucieux de ne pas perdre de la place dans le paysage audiovisuel européen. Produit d'un projet politique d'intégration et de diffusion de la culture, soutenue par un projet culturel de légitimation, Arte trouve dans la technologie le moyen de réalisation des fins politiques de sa création. Le satellite, la numérisation de la diffusion télévisuelle, les nouvelles possibilités techniques de transmission du son et de l'image vont élargir le champ d'action de son slogan de «culturelle européenne » et provoquer un effet rétroactif sur les fins du projet politique, en lui ajoutant des nouvelles perspectives. Articulée sur trois logiques - politique, culturelle et technique - la chaîne affirmera un profil où la créativité reste la marque européenne fondamentale, et les 
possibilités techniques de couverture des satellites ajoute au partenariat franco-allemand un élargissement de la marge de manœuvre de son action dans le paysage audiovisuel.

\section{Culture du Monopole}

2 C'est après 23 ans de monopole que l'État prend la décision de replacer la culture et l'éducation dans l'esprit du service public en donnant naissance en premier à la SEPT (Société Européenne de Programmes de Télévision), qui n'était au début qu'un centre de production de programmes et, plus tard, finit par se transformer en une véritable chaîne de diffusion avec sa propre grille de programmes. En alliance avec l'Allemagne, la France signe donc l'accord interétatique qui officialise face à l'Union Européenne, l'existence de la chaîne. Cet accord est l'une des stratégies de légitimation du projet politique d'Arte qui, pour exister et porter le slogan de "culturelle européenne » ne pourrait être un « fait " d'un seul pays. Arte est issue d'un accord interétatique réglementé comme un groupement européen d'intérêt économique, le GEIE, forme de réglementation qui permet à la société d'être indépendante des lois nationales des deux pays partenaires. Le GEIE, dans le cas d'Arte, est une voie de concrétisation de la stratégie d'alliance et coopération entre les deux pays, puisque les réglementations française et allemande dans l'audiovisuel sont assez différentes, voire opposées. Si le système audiovisuel français est fondamentalement centralisé et contrôlé par des organismes du service public, l'Allemagne a un système de complète décentralisation. Le pouvoir public ne peut imposer ou décider des règles pour la télévision, qui est sous le contrôle des Länder et des membres de la société civile, ce qui permet à la télévision allemande une régionalisation plus intense que celle pratiquée en France.

Créer Arte et la légitimer comme porteuse de la mission de l'État Français de faire survivre la culture, en lui ajoutant une valeur européenne, bien ancrée dans les efforts de création d'une Europe, peut être entendu comme une stratégie de l'État pour non seulement se maintenir dans cet espace où il perd une place considérable avec les privatisations, mais aussi pour faire parvenir et justifier sa mission de promoteur de culture et de programmes de qualité.

Dans cet axe, on retrouve les points principaux qui constituent les moyens par lesquels cette chaîne va réussir à justifier son existence. Le premier de ces points est évidemment l'accord signé avec l'Allemagne pour les recherches concernant les satellites, point clé du projet politique qui prévoit déjà la rétroaction technologique. Ensuite, l'accord de création de la chaîne, suivi de sa mise en pratique avec, une fois de plus, les partenariats de production et coproduction et l'utilisation des nouvelles technologies comme un des instruments qui lui permet de mener une faible mais réelle concurrence avec les chaînes privées. Cela va aussi permettre à l'État de maintenir le doigt bien placé sur les questions concernant l'occupation de l'espace audiovisuel français. Arte peut figurer ici comme un axe de stratégie du service public audiovisuel en France, qui compte déjà avec ses chaînes généralistes (France 2 et France 3). Celles-ci, étant donné ces caractéristiques généralistes, sont évidemment plus en état de concurrence avec les chaines privées, puisqu'elles adoptent le même mode de transmission et la même structure de programmation que ces dernières. Mais Arte prime par la différence, le partage et la diversité, cette dernière occupant à son tour un rôle stratégique dans l'opposition cachée et masquée au modèle américain de production audiovisuelle. Elle naît avec un futur quasi garanti, légitimé par son caractère culturel et la préoccupation majeure avec la 
qualité de sa production, libre des contraintes publicitaires et entièrement financée par le service public.

\section{La technique comme stratégie}

5 Arte est née pour marcher main dans la main avec les nouvelles technologies. Cette stratégie, ou un des moyens de la fin politique, a permis que la chaîne puisse porter son slogan de culturelle et européenne, ainsi que figurer dans le marché audiovisuel de l'Europe comme une chaîne de présence marquante. Cette présence est loin d'être évidente si la méthode d'évaluation se base sur les taux d'audience d'Arte, presque effacés à côté des chiffres des chaînes généraliste. Mais la grandeur du projet politique et son ambition placent la chaîne dans un paysage aussi large que celui couvert par ses concurrentes : celui de la culture européenne. La croissance des taux d'audience, qui doivent ici être isolés des autres taux du paysage audiovisuel européen, résulte des trois facteurs qui sont à la genèse d'Arte : politique, culture et technologie. La rétroaction de cette dernière sur le duo volonté politique/projet culturel présente déjà ses résultats. Les taux d'audience montrent une croissance de 2,5\% au cours des sept ans d'existence de la chaîne, qui pendant le premier trimestre de 1999 a atteint 3,5\% en France et 0,7\% en Allemagne.

6 Aujourd'hui, la chaîne occupe une place considérable dans l'audiovisuel de l'Europe : à jour avec les nouvelles technologies de diffusion. Au moment où les discussions pour la mise en place du numérique hertzien viennent envahir ce paysage en mettant en jeu la concurrence entre les opérateurs de télédiffusion par satellite, l'audience et la production audiovisuelle, Arte peut se trouver face à un scénario décisif pour augmenter encore plus sa présence dans le paysage audiovisuel européen. L'occupation d'un canal de diffusion sur le réseau numérique hertzien va accroître son public potentiel.

7 Certains acteurs du paysage audiovisuel européen suggèrent même que la seule manière de faire concurrence à la domination américaine dans le marché européen est de susciter une attitude de renforcement de la télévision européenne, ce qui consiste à mettre en place des chaînes culturelles européennes, des normes de diffusion ainsi que le projet de la télévision à haute définition. Cela consiste, en partie, à numériser la télévision dans sa totalité, c'est-à-dire permettre tant au public câblé qu'au public qui reçoit encore la diffusion hertzienne, d'accéder au numérique. Et cela semble possible maintenant que les normes de mise en place du numérique hertzien commencent à être définies.

\section{Un projet culturel}

8 S'imposer dans ce marché dépend donc de la construction et des stratégies adoptés par la chaîne pour assumer le slogan qu'elle porte dès sa naissance; celui de chaîne culturelle européenne. L'alimentation créative du projet culturel passera par une logique technologique qui va élargir le champ d'action de la culture proclamée par Arte. Cette logique technologique figure comme un des moyens de la concrétisation des fins du projet politique. Les possibilités techniques de la diffusion satellitaire élargissent le champ d'action de la culture européenne d'Arte dans la mesure où la diffusion peut se donner à une échelle plus large, ainsi que les possibilités de l'utilisation de plusieurs canaux dans un système numérique. Le projet culturel figure donc ici comme une toile de 
fond d'une initiative française qui trouve sa légitimation dans l'idée d'une Europe en construction. Ces trois mots, stratégie, culturelle et européenne se rencontreront dans toutes les démarches de la construction du profil de la chaîne. Soit dans l'opportunisme de son lancement en même temps que les satellites sont mis en orbite et le paysage audiovisuel est redéfini, soit dans le mode de fonctionnement administratif et l'élaboration et production de sa grille de programmes.

9 Les innovations les plus récentes et l'impact des nouvelles technologies sur la diffusion des images ont, d'une certaine manière, permis l'apparition d'Arte dans le paysage audiovisuel européen. En considérant l'hétérogénéité et la diversité culturelle de cet espace, la chaîne a été créée pour atteindre un public justement non-uniforme en ce qui concerne les références culturelles. Elle devra donc faire usage d'une stratégie de sensibilisation de ce public, puisque son outil premier est la culture, et une culture qui n'est pas la même partagée par tout son public potentiel. Insérée dans une réalité planétaire, dans un temps où l'audiovisuel devient de plus en plus mondialisé avec des caractéristiques plutôt généralistes, Arte s'impose comme un instrument de promotion de la culture européenne; et c'est exactement ce caractère qui va lui permettre de construire un profil capable d'atteindre une échelle européenne de diffusion en bénéficiant de la technologie de télétransmission par satellite. Cette technologie apporte la possibilité de l'utilisation des nouveaux modes de consommation audiovisuelle qui vont accentuer la concurrence dans tous les champs de la production audiovisuelle. Ainsi, la maitrise et la mise en place de ces outils va permettre à la chaîne de concurrencer les grands groupes privés dans des domaines qui ne sont pas la télévision, mais les produits parallèles comme les sites Internet, les CD Rom et les publications. Mais les enjeux politiques et économiques autour de la maintenance d'Arte dans cet espace sont plutôt difficiles à supporter. Pour que les chaînes publiques, d'une manière générale, se maintiennent dans le marché, il est évidemment nécessaire que l'État intervienne à des degrés variés sur la réglementation de ce marché. Et une des stratégies pour l'occupation de cet espace se révèle dans la signature des accords avec les groupes privés qui occupent déjà le marché, soit dans le sens d'exploiter les technologies, soit dans le sens de coproduire avec les systèmes de partenariat.

Cela est loin de consister en un simple protectionnisme et semble plus un investissement, au contraire par exemple de la réglementation pour la publicité et le système des quotas. Dans le cas d'Arte, les ressources de la chaîne viennent en grande partie de subventions d'État, des accords de coproduction et de la redevance. N'ayant pas de publicité, Arte est porteuse, d'une certaine manière, d'un privilège légitimé par sa fonction d'instrument public de défense de la culture européenne. Mais quelle culture européenne? Est-ce possible que deux pays proposent une démonstration de cette culture? Ou cette démonstration ne serait-elle qu'une « vision » de la culture européenne?

11 Une des hypothèses que nous proposons dans ce travail est que la culture tant célébrée du slogan d'Arte est européenne dans la mesure où elle est la «vision » française de cette culture. Et plus française qu'allemande. Et cela est le résultat non seulement de la motivation qui a mené à la création de la chaîne, motivation partie de la France, mais aussi de la manière selon laquelle les deux pays conçoivent et administrent la production télévisuelle. Au sein de la chaîne, des entretiens avec le personnel du service international ont pu révéler que ceux-ci voient l'initiative de production et de création de films et documentaires comme un fait des acteurs français d'Arte. Si les thèmes des documentaires et les metteurs en scène des films proposés sont européens, voire africains 
ou asiatiques, les choix restent en grande partie français. En Allemagne, la production des programmes destinés à Arte est réalisée par des équipes engagées aussi avec les autres chaînes du service public. Et « ce sont des gens qui s'occupent d'Arte mais qui s'occupent aussi de programmes pour leurs propres chaînes ", explique le directeur du service des relations internationales de la chaîne à Paris. Si du côté français la notion de centralisation est un fait, pour l'Allemagne, avec son système audiovisuel décentralisé, cela n'est pas valable. "Nous sommes tous rassemblés ici, c'est notre seule mission " affirme le directeur de l'unité relations Internationales. Exporter la culture européenne dans le continent et au delà des frontières européennes à travers Arte peut donc gérer une confusion quant à l'origine du produit, qui est fondamentalement français.

\section{Technologie}

12 La création d'Arte s'inscrit dans une logique technologique qui a occupé le paysage audiovisuel européen dès que les premiers satellites de télédiffusion commencèrent à être lancés et changèrent la dynamique de diffusion de l'information.

13 La possibilité de transmission de l'image et du son par satellite est responsable d'une vraie révolution dans la télévision, non seulement européenne, mais mondiale. En même temps le scénario des évolutions technologiques change à une vitesse considérable avec les recherches concernant le perfectionnement des moyens de transmission de l'information dans le monde entier.

Les avantages de la transmission par satellite et l'utilisation des nouvelles technologies en ce qui concerne équipements et production ont été au sein de la conception d'Arte et on permis la construction du profil actuel de la chaîne. La logique technologique qui soustend les projets politiques et culturels finit par modifier les fins du premier. Au delà du partenariat France-Allemagne, la chaîne a intégrée un paysage plus large, ce qui permet de justifier l'adjectif européenne présent dans son slogan. C'est grâce aux techniques de diffusion par câble et satellite qu'Arte peut être aussi reçue au-delà de l'axe nord/sud de l'Europe.

15 Les partenariats et l'exportation de programmes diffusés dans les pays du l'Est européen, possibles à travers plusieurs accords signés avec la Hongrie, la Roumanie, la Russie, la Bulgarie, la République Tchèque, la Pologne et la Slovaquie entre 1990 et 1997, sont le fait d'une rétroaction de la technologie sur le projet politique initial. Le rapprochement et la compréhension entre les peuples préconisés dans le traité de création devient un fait par le biais de la technologie qui lie ces peuples en réseaux ${ }^{1}$. Le public, en Europe Centrale ou de l'Est, a sa régularité et, lui aussi, son profil construit. Arte a su en tirer partie et s'identifier avec la demande de ces pays. L'accord de coopération date de 1990 et assure la diffusion mensuelle sur les chaînes des pays cités de plus de dix heures de programmes produits par Arte. En échange, la chaîne franco-allemande reçoit aussi des programmes produits par les chaînes qui ont passé l'accord. Ces programmes sont rediffusés par Arte sur ses réseaux hertziens.

La demande croissante du public d'Europe centrale a fait que ces accords sont renouvelés chaque année, ce qui a entrâné l'aide du Ministère des

Affaires Etrangères avec des subventions annuelles qui tournent autours de deux millions de francs. En constatant cette demande des pays d'Europe Centrale, Arte a proposé une série de programmes adaptés aux habitudes télévisuelles de ces pays. L'observation de la 
grille de programmes de ces chaînes montre que la demande peut être bien différente de celle de l'Europe de l'Ouest. Entre 1992 et 1995, 48 programmes de musique - y compris présentation d'orchestre, concerts et opéras - ont été incorporés à Arte en provenance des télévisions tchèque, hongroise, polonaise et de Saint-Pétersbourg. Arte n'a pas imposé une grille mais étudié la demande de ces pays et proposé des options de coproduction, augmentant ainsi sa propre banque de programmes. Le paysage audiovisuel de l'Est de l'Europe est dans un moment délicat. Après la chute du système socialiste, ces pays se sont retrouvés face aux enjeux qui agitaient l'audiovisuel mondial. Le marché des pays comme la Hongrie, la Russie, la Roumanie et la Pologne, était un espace encore vierge et évidemment les regards des grands groupes de télécommunication et de télévision se sont vite tournés vers eux. L'alternative américaine avec ses chaînes thématiques qui font sauter les chiffres de l'audimat représentait, bien sûr, une menace. Arte a été la première à passer des accords avec les chaînes de l'Europe de l'Est, avec une stratégie de pénétration de marchés basée sur l'introduction des coproductions pratiquement "personnalisées ", c'est-à-dire avec des programmes capables de provoquer une identification nationale dans chaque pays, ce qui finit par affirmer et confirmer l'image de marque de la chaîne. Un simple exemple est le "paquet » de programmes échangés avec la télévision de Saint-Pétersbourg entre 1992 et 1995. La danse classique étant l'activité culturelle traditionnelle de cette ville, la chaîne russe a fourni à Arte 30 programmes de danse, entre spectacles et documentaires. Toujours en plaçant la culture à côté des rapports commerciaux, Arte a choisi une stratégie de niche dans ce marché à travers la construction d'un lien de confiance. Au lieu de proposer les programmes dans des catalogues, la chaîne "franco-allemande" laisse le chemin libre pour que ces télévisions puissent choisir les programmes à diffuser. En retour, Arte bénéficie de l'exclusivité de droits pour deux ans sur la diffusion des programmes obtenus de l'échange, ce qui permet aussi le retour sur investissements.

Mais il est important de signaler que la diffusion à échelle européenne n'est pas un argument suffisant pour considérer une télévision comme porteuse d'une identité européenne. Attribuer à la capacité de diffusion à large échelle, grâce au satellite, le titre d'identité européenne comporte le risque de réduire cette identité à une simple question de territoire physique. Et là, les stratégies d'Arte sont claires: la différence sera l'instrument pour trouver dans ce public dit européen des éléments de références qui puissent parvenir à créer le lien social nécessaire à l'existence d'une chaîne publique, gratuite et à la portée de tout citoyen européen. La caractéristique européenne n'est donc pas liée au fait que la diffusion se donne dans toute l'Europe, mais au fait que le contenu de cette diffusion intéresse et soit suffisamment attractif pour attirer l'attention du public européen. Le projet d'entreprise du groupe Sept/Arte et La Cinquième pour 1999-2001 est clair : participer à la " construction du lien social, en créant un espace de communication et de débat » est un des objectifs, apparu timidement au moment de la création de la chaîne et maintenant repris comme point important à la constitution et affirmation de son profil. Ce point est peut être le plus polémique du projet du groupe. Dès sa création, il a été vu comme l'initiative qui permettrait la mise en place du rêve de la télévision sans frontières, européenne et culturelle. Cependant, les critiques à ce concept de télévision sont nombreuses.

La constitution d'un "ghetto culturel » dans la télévision française et le manque de légitimité d'une télévision publique à faible potentiel d'audience sont des débats qui reviennent à chaque discussion budgétaire pour définir le futur des subventions. La 
numérisation du réseaux hertzien ne risque que d'augmenter cette constante remise en cause. Si le service public est destiné à tous les citoyens et tous les citoyens ne regardent pas Arte à $100 \%$, pourquoi la maintenir pour une élite culturelle? Ou alors, pourquoi ne pas la faire devenir une chaîne payante? Ici les trois logiques présentées au début de ce texte affirment leur interaction.

Dominique Wolton, dans un article publié dans L'Express² ${ }^{2}$ u 29 octobre 1993 soulignait le doute concernant la segmentation d'un public sélectif pour l'audience d'Arte. Pour Wolton, les spécifications conçues pour la chaîne dès sa création motivaient une sélection de public ; Arte, pour lui, était destinée à une couche sociale intellectualisée. «(...) la création d'une chaîne de ce type me semble contraire à l'idée que j'ai de la télévision, du moins telle qu'elle devrait exister dans une démocratie moderne comme la France, c'està-dire sous la forme d'un moyen de communication destiné à tous, et pas seulement à un public spécialisé ». Même si les chiffres de l'audience d'Arte pendant ses premiers jours contestaient déjà la position de Wolton, restait toujours le fait que la chaîne avait un public trop réduit pour une télévision publique. Dans Télévisions Publiques en quête d'Avenir, Yves Achilles rappelle que dans les études réalisées par la SOFRES l'audience d'Arte ne se concentre pas dans une seule niche de la population mais se trouve un peu éparpillée sur plusieurs segments de la population, soit professionnels libéraux, cadres, étudiants ou populations des régions écartées des grands centres urbains et, principalement, les ouvriers qui constituent un public plus nombreux que celui constitué par les cadres. Achilles signale que "un tiers de l'audience réside en milieu rural dans les agglomérations de moins de 20000 habitants $»^{3}$.

21 Cette mise en cause trouve encore un fort soutien dans le fait que les supports budgétaires de la chaîne deviennent de plus en plus élevés. L'État peut-il, va-t-il continuer à destiner ces subventions à Arte ? Le revenu est faible et l'audience (1 à $2 \%$ en 1993 et 3,5 \% en 1998) n'est pas en mesure de faire concurrence aux chaînes privées commerciales gratuites. 73 \% de l'argent consacré à la chaîne dépend des subventions budgétaires de l'État. Ce montant provient des recettes gérées par les privatisations, qui à leur tour dépendent des variations du contexte économique et financier autour de ce secteur.

Les incertitudes autour d'Arte sont aussi liées aux questions posées quand aux modes de transmission de la chaîne et la création d'un public réellement européen. Si en Allemagne Arte a sa place dans le réseau câblé, en France sa diffusion reste hertzienne, mais avec quelques expériences dans le champ du numérique et de l'analogique. Le mode de diffusion hertzien a déjà été remis en cause par la commission des finances du Sénat français qui a proposé, en 1993, de réduire le montant destiné à Arte (350 millions de francs sur le budget total d'un milliard de francs ${ }^{4}$ ). Mais il fait partie d'une stratégie bien simple qui ne peut se passer d'observer le contexte de l'audiovisuel français. Si en Allemagne $70 \%$ des foyers sont câblés, en France la réalité n'est pas la même. L'histoire de la télévision française s'est toujours faite par voie hertzienne. Le marché télévisuel français a vu l'arrivée des câbles et des chaînes satellites bien en retard par rapport à la rapidité de ce même phénomène en Allemagne : à peine $13 \%$ des foyers français sont équipés de câbles. Il est donc naturel que, même en disposant de la technologie numérique et analogique, la transmission d'Arte en France se fasse par voie hertzienne, en dépit du coût de ce genre de transmission, qui demeure assez élevé par rapport à la diffusion par câble ou satellite.

Ensuite, la conjoncture actuelle avec les discussions autour de l'implantation du numérique hertzien comme substitution à toute transmission analogique remet en cause 
l'existence, ou du moins le caractère de service public, d'Arte. La technologie de transmission du numérique par voie hertzienne peut encore une fois modifier l'usage de la télévision en clair. La télévision de proximité est l'un des points les plus discutés au sujet de la numérisation du réseau hertzien. Le multiplexage multiplie le nombre de canaux et permet l'augmentation à coûts réduits des canaux de diffusion. La tendance serait donc, pour la télévision publique à une décentralisation avec la création des télévisions locales. Qu'en serait-il d'Arte dans ce paysage? Sa mission de service public pourrait être remise en cause.

\section{Quel public ?}

Si l'idée «franco-allemande » de " télévision européenne » n'a pu voir le jour que grâce aux progrès techniques des moyens de diffusion, la notion d'existence d'un "public européen » n'était pas évidente. La constitution d'une chaîne destinée à un public qui, au delà des différences d'âge ou de sexe, se trouve partagé en plusieurs cultures et langues différentes suppose une large appréciation de ces différences. Il est inévitable de poser la question : existe-t-il vraiment un public européen? Et comment trouver ce public. Parler d'une identité culturelle européenne paraît illusoire quand on observe la diversité culturelle présente dans les pays de ce territoire. Cette diversité joue son rôle dans le projet culturel dont Arte est le fruit. C'est la mosaïque culturelle qui fait la stratégie de la différence ; stratégie dont la chaîne se sert pour justifier sa logique politique.

\section{BIBLIOGRAPHIE}

ACHILLES Yves, Les télévisions publiques en quête d'avenir, Presses Universitaires de Grenoble, Grenoble, 1994, 327 p.

BARTHES Roland, Mythologies, Éditions du Seuil, 1957, 233 p.

BAUDELOT Philippe et EYMERY Gérard, Les satellites et l'audiovisuel, Éditions Dixit, Paris, 1994, $292 \mathrm{p}$.

FUMAROLI Marc, L'État culturel : essai sur une religion moderne, Éditions de Fallors, Paris, 1992, $411 \mathrm{p}$.

HERMANUS A-Merry, Tempêtes sur l'audiovisuel, Éditions du Perron, Liège, 1990, 247 p.

HUBER Richard, La RFA et la télévision, INA/Champ Vallon, Paris, 1988, 142 p.

ISAR Hervé, Le service public et la communication audiovisuelle, Presses Universitaires d'Aix-

Marseille, 1995, 409 p.

JEANENEY Jean-Noël, Une histoire des médias, Éditions du Seuil, Paris 1996, 375 p.

LABIT Claude, La télévision du futur, Paru dans Le Courrier du CNRS, Dossier Scientifique Signaux et images, n $^{\circ} 77$, juin 1991, pp 47. 
MARTELAIT Armand et PIEMME Jean-Marie, Télévision : enjeux sans frontières, Presses Universitaires de Grenoble, Grenoble, 1980, 229 p.

MATTELART Armand, L'Invention de la communication, La Découverte, Paris 1994, 376 p.

MIEGE Bernard, PAJON Patrick et SALAM Jean-Michel, L'industrialisation de l'audiovisuel, Éditions Alibier, Paris, 1986, 284 p.

MOLLARD Claude, L'Ingénierie culturelle, Que sais-je ? Presses Universitaires de France, Paris, 1994, $128 \mathrm{p}$.

MORIN Edgar, Penser l'Europe, Gallimard, Paris, 1987, 265 p.

MUCCHIELLI Alex et GUIVARCH Jeannine, Nouvelles méthodes d'étude des communications, Armand Colin, 1998, $174 \mathrm{p}$.

MUSSO Pierre, SOUETRE Philippe et LEVASSEUR Lionel, Presse écrite et télévision dans les régions d'Europe, Éditions du Conseil de l'Europe, Pays Bas, 1995, 245 p.

RABATE François, Diffusion hertzienne, Paru dans Dossiers de l'audiovisuel, n 57, décembre 1994, IN A/La documentation française, pp 31.

SFEZ Lucien, Critique de la communication, Éditions du Seuil, 1988, 400 p.

UTARD Jean-Michel, Arte : Information télévisé et construction d'un point de vue transnational. Etude d'un corpus franco-allemand, Thèse de Doctorat en Sciences de l'Information et Communication, Strasbourg, 1997, 347 p.

VEDEL Thierry, Les techniques de communication : voie hertzienne, satellite, câble, Paru dans Les Médias - Cahiers Français, Ed. La Documentation Française, n² 266, Paris, mai/juin 1994, pp 3.

WOLTON Dominique et Missika Jean-Louis, La folle du logis : la télévision dans les sociétés démocratiques, Éditions Gallimard, 1983, 338 p.

WOLTON Dominique, Eloge du grand public : une théorie critique de la télévision, Flammarion, Paris 1990, $315 \mathrm{p}$.

WOLTON Dominique, Penser la communication, Flammarion, Paris, 1997, 402 p.

\section{NOTES}

1. Utard, Jean-Michel. Arte : information télévisée et construction d'un point de vue transnational. Etude d'un corpus franco-allemand. Thèse pour le doctorat Nouveau Régime en Sciences de l'Information et de la Communication, sous la direction de Yves Lavoine, Univ. Robert Schuman, Strasbourg III, 1997. 349 pages. Page 66.

2. Source : Achilles, Yves. Les Télévision Publiques en Quête d'Avenir

3. Ibid 1 , page 267

4. Source : Achilles, Yves Les Télévisions Publiques en Quête d'Avenir 


\section{RÉSUMÉS}

Ce travail a été développé dans le cadre d'un DEA en Information et Communication à l'Université de Bordeaux 3 pendant l'année 1998-1999. La problématique centrale est celle de l'interaction de trois logiques - politique, culturelle et technologique - pour la construction et l'expansion de la chaîne culturelle franco-allemande Arte. D'abord comme un projet politique, légitimé par le projet culturel, la chaîne prend une dimension européenne qui n'est possible qu'en fonction de l'utilisation des nouvelles technologies dans l'audiovisuel. Cette technologie, comme un moyen d'obtention des fins politiques du projet, rétroagit sur celui-ci et élargit le champ d'action européen de la diffusion d'Arte.

This thesis was developed during the course of a DEA in Information and Communication in the University of Bordeaux 3 during 1998-1999. The central question is the interaction of three topics - politics, culture and technology - for the construction and expansion of French-German cultural channel Arte. As an politic project, made genuine by the cultural project, the channel has an Europeen dimension only made possible by using new technologies in the audiovisual field. This technologies, used to obtain the political goal of the project, retrains itself and at the same time enlarges the Europeen range of Arte's diffusion.

\section{AUTEUR}

\section{NAHIMA VIANNA}

Nahima Vianna une thèse au LABCIS, laboratoire que dirige Pierre Fayard à l'Institut de la Communication et des Nouvelles Technologies de l'Université de Poitiers. Elle travaille sur les nouvelles technologies, l'audiovisuel et la diffusion en télévision. 